INPLASY

PROTOCOL

To cite: Xu et al. A metaanalysis of randomized controlled trials of virtual reality exposure therapy for posttraumatic stress disorder. Inplasy protocol 202080083. doi:

10.37766/inplasy2020.8.0083

Received: 19 August 2020

Published: 20 August 2020

Corresponding author:

Ran Wang

wangran104@hotmail.com

Author Affiliation:

The First Hospital of Hebei

Medical University

Support: National Natural

Fund

Review Stage at time of this submission: Data analysis.

Conflicts of interest:

No Conflicts of interest.

\section{A meta-analysis of randomized controlled trials of virtual reality exposure therapy for post-traumatic stress disorder}

Xu, S1; Wan, J2; Wang, X33 Wang, R4.

Review question / Objective: To systematically evaluate the effects of virtual reality exposure therapy (VRET) in the treatment of post-traumatic stress disorder (PTSD).

Condition being studied: Post-traumatic stress disorder (PTSD) is a kind of mental illness with severe clinical symptoms and great damage to mental health.In the treatment of PTSD, the efficacy of exposure therapy has been supported by a number of clinical studies.At the same time, virtual reality (VR) has made great progress in recent years.VR technology can deliver vision, hearing and various senses to users through headphones, making them feel like they are in a virtual or imaginary environment.The virtual reality-based exposure therapy (VRET), which is produced by the combination of exposure therapy and virtual reality technology, provides a new inspiration for solving the limitations of exposure therapy in the treatment of PTSD.

INPLASY registration number: This protocol was registered with the International Platform of Registered Systematic Review and Meta-Analysis Protocols (INPLASY) on 20 August 2020 and was last updated on 20 August 2020 (registration number INPLASY202080083).

\section{INTRODUCTION}

Review question / Objective: To systematically evaluate the effects of virtual reality exposure therapy (VRET) in the treatment of post-traumatic stress disorder (PTSD).
Condition being studied: Post-traumatic stress disorder (PTSD) is a kind of mental illness with severe clinical symptoms and great damage to mental health.In the treatment of PTSD, the efficacy of exposure therapy has been supported by a number 
of clinical studies.At the same time, virtual reality (VR) has made great progress in recent years.VR technology can deliver vision, hearing and various senses to users through headphones, making them feel like they are in a virtual or imaginary environment.The virtual reality-based exposure therapy (VRET), which is produced by the combination of exposure therapy and virtual reality technology, provides a new inspiration for solving the limitations of exposure therapy in the treatment of PTSD.

\section{METHODS}

Participant or population: Participants had to be aged 18-60, have PTSD based on Diagnostic and Statistical Manual of Mental Disorders, 4thed.(DSM-IV) criteria and confirmed by structured interview. Participants were excluded if they were actively suicidal, homicidal, psychotic, or suffering from substance dependence that was not at least in early remission.

Intervention: Virtual reality-based exposure therapy are the main intervention.

Comparator: Control group received traditional treatment or in waitlist.

Study designs to be included: Randomized controlled trials(RCTs) will be included.

Eligibility criteria: The type of study was a randomized controlled study, regardless of whether the study was blind or not, with the restricted language being English;The subjects were human beings; Posttraumatic stress disorder was the disease targeted in the study.

Information sources: Databases including Pubmed, Cochrane Library, and EMbase were searched by computer.

Main outcome(s): The CAPS scale score was used as the criterion for the severity of PTSD symptoms to analyze and compare the efficacy of VRET and traditional treatment for PTSD symptoms The normalized mean difference (SMD) was used as the effect index, and the point estimate and $95 \%$ confidence interval were given for each effect.

Quality assessment / Risk of bias analysis: Two reviewers will independently assesses the quality of the selected studies according to the Cochrane Collaboration's tool for randomized controlled trials. Items will be evaluated in three categories: Low risk of bias, unclear bias and high risk of bias. The following characteristics will be evaluated: Random sequence generation (selection bias) Allocation concealment (selection bias) Blinding of participants and personnel (performance bias) Incomplete outcome data (attrition bias) Selective reporting(reporting bias) Other biases Results from these questions will be graphed and assessed using Review Manager 5.3.

Strategy of data synthesis: All analyses were performed using RevMan 5.3 and Stata 15.0 software.The results in this paper are all measurement data. Since the mean difference between the treatment group and the control group is large, the standardized mean difference (SMD) is used as the effect index, and the point estimate and $95 \%$ confidence interval of each effect amount are given.The $\times 2$ test was used to analyze the heterogeneity of the included studies, and the studies without heterogeneity ( $P>0.1,12<50 \%)$ Fixed effect model can be used for combination analysis; In the presence of heterogeneity $(P<0.1,12>50 \%)$, the random-effects model was used.According to the possible factors of heterogeneity, the method of sub-group analysis is adopted to analyze the reasons for the existence of heterogeneity, the Egger method is used to test publication bias, and the sensitivity analysis is used to test the stability of the results.P $<0.05$ was considered statistically significant.

Subgroup analysis: We will consider subgroups such as differences treatment methods between traditional treatment groups, clinic type, and location(rural/ urban). 
Sensibility analysis: Sensitivity analyses were performed using a case-by-case exclusion analysis for the efficacy of VRET in the treatment of overall PTSD symptoms. Country(ies) involved: China.

Keywords: Virtual reality exposure therapy; Post-traumatic stress disorder; Meta analysis.

Contributions of each author:

Author 1 - Shuqi Xu.

Author 2 - Jinling Wan.

Author 3 - Xueyi Wang.

Author 4 - Ran Wang. 